\title{
Is grafting biomaterials or biological agents more effective than open-flap debridement in treating deep intraosseous defects?
}

\author{
Is the use of grafting biomaterials or biological agents and open-flap \\ debridement (OFD) more effective than OFD alone for treatment of deep \\ intraosseous defects?
}

\begin{abstract}
Trombelli L, Heitz-Mayfield L, Needleman I, Moles D, Scabbia A. A systematic review of graft materials and biological agents for periodontal intraosseous defects. J Clin Periodontol 2002; 29(Suppl. 3):S117-S135
\end{abstract}

Data sources Sources were MEDLINE and the Cochrane Oral Health Group Trials Register. Reference lists from relevant articles, and selected journals dated up to April 2001, were also searched by hand.

Study selection Randomised controlled trials (RCT) were selected if they were of at least 6 months' duration and they compared the grafting of biomaterials or biological agents - alone or in combination - plus OFD (including open flap curettage, access flap surgery, modified Widman flap) to OFD alone or in combination with a placebo. Any type of grafting biomaterials or biological agents, apart from guided tissue regeneration, alone or in combination with other biomaterials/biological agents were considered.

Data extraction and synthesis Information regarding the quality and characteristics of studies were extracted independently by two reviewers. Short-term, long-term and a range of patient-centred outcomes were assessed. The main short-term outcomes were the changes in clinical attachment levels (CAL) and probing pocket depth (PPD). Long-term measures included disease recurrence - measured as the proportion of defects presenting loss of CAL and/or bone, as assessed clinically or radiographically from 12 months after intervention - and the change of CAL from 6-12 months up to the last observation interval. A weighted treatment effect was calculated and the results were expressed as weighted mean differences for continuous outcome variables using both fixed and random models. The analysis for the continuous outcome variables was conducted using Stata (version 6; Stata Corporation, College Station, Texas, USA) to combine parallelgroup and intra-individual (split-mouth) studies.

Results The difference in CAL change between test and control groups varied from $-1.45 \mathrm{~mm}$ to $1.40 \mathrm{~mm}$ with respect to different biomaterials or biological agents. Meta-analysis showed that CAL significantly improved after treatment with coralline calcium carbonate (weighted mean difference, $0.90 \mathrm{~mm} ; 95 \%$ confidence interval $(\mathrm{Cl})$, $0.53-1.27$ ), bioactive glass (weighted mean difference, $1.04 \mathrm{~mm} ; 95 \%$ $\mathrm{Cl}, 0.31-1.76$ ), hydroxyapatite (weighted mean difference, $1.40 \mathrm{~mm}$; $95 \% \mathrm{Cl}, 0.64-2.16$ ) and enamel matrix proteins (weighted mean difference, $1.33 \mathrm{~mm}, 95 \% \mathrm{Cl}, 0.78-1.88$ ). Heterogeneity in the results between studies was highly statistically significant, however, for most biomaterials or biologicals; this could not be fully explained.

Conclusions Overall, the use of specific biomaterials or biological agents was more effective than OFD in improving attachment levels in intraosseous defects. Difference in CAL gain varied greatly with respect to different biomaterials/biological agents. Because of the significant

Address for correspondence: Leonardo Trombelli, Research Centre for the Study of Periodontal Diseases, University of Ferrara, Corso Giovecca, 203-44100 Ferrara, Italy. E-mail: I.trombelli@unife.it heterogeneity in results between studies in most treatment groups, general conclusions about the clinical benefit of graft biomaterials/ biologicals need to be interpreted with caution.

\section{Commentary}

The authors should be commended for their attempt to bring order to the chaos of studies of various graft materials and biological agents for the treatment of intra-osseous defects. The review shows that at least 18 distinctly different treatments were tested in 26 RCT that fulfilled the inclusion criteria. Most of the included studies have been of a rather short duration; have comprised rather few patients; and are characterised overall by considerable heterogeneity with respect to their design, methods, organisation, outcomes and maintenance-treatment schemes.

The authors conclude that the use of specific biomaterials/ biologicals was more effective than OFD in improving the attachment levels of intra-osseous defects. They also note a significant heterogeneity between studies in most treatment groups, however, which necessitates a cautious interpretation of the possible clinical benefits of these biomaterials.

Although we agree with the latter statement, we find it difficult to support the conclusion about the beneficial effect of certain treatments that were investigated. The authors note that out of the $26 \mathrm{RCT}$, only $10(38 \%)$ presented an adequate randomisation method; methods for allocation-concealment were adequate in only five trials (19\%); blinded outcome assessment was reported in seven trials (27\%); and therapists were blinded to treatmentassignment in only two trials (8\%). These results again demonstrate the preponderance of poor-quality RCT in periodontology, as reported recently by Montenegro et al. ${ }^{1}$ We were able to deduce that only one study ${ }^{2}$ was found to fulfil all four quality criteria in the present review. All 26 RCT, however, were included in the analyses despite the fact that low-quality RCT are notoriously prone to bias and meta-analyses do not turn poor-quality studies into high-quality trials. We are therefore concerned that a review such as this may be interpreted as proof that the evidence has been carefully sifted, the reader stopping further scrutiny after reading the abstract statements that, "CAL change significantly improved after treatment with substance $\mathrm{X}, \mathrm{Y}$, and Z."

One of the treatment modalities included in the present review is enamel matrix proteins, and this provided us with the opportunity to make a comparison with the recent Cochrane review of enamel matrix proteins. ${ }^{3}$ Indeed, interesting differences were noted. Hence, the only "high quality" study of the present review was scored as being not blinded with respect to the outcome assessors in the Cochrane review. The study of ${ }^{4}$ was excluded from the present review on the grounds of the defect/site, not the patient, being the statistical unit used; while it could be included in the Cochrane review. These disparities demonstrate that although systematic reviews add transparency to the review process, they do not provide 
a safeguard against biases, as high quality periodontal RCT's are all too few.

\section{Practice point}

- Currently there is no evidence to support the use of these graft materials and biological agents for periodontal intra-osseous defects.

\section{Rodrigo Lopez, Vibeke Baelum}

Department of Community Oral Health and Paediatric Dentistry, Faculty of Health Sciences, University of Aarhus, Aarhus,

Denmark
1. Montenegro R, Needleman I, Moles D, Tonetti M. Quality of RCTs in periodontology - a systematic review. J Dent Res 2002; 81:866-870.

2. Tonetti MS, Lang NP, Cortellini $P$ et al. Enamel matrix proteins in the regenerative therapy of deep intrabony defects. J Clin Periodontol 2002; 29:317-325.

3. Esposito M, Coulthard P, Worthington HV. Enamel matrix derivative $\left(\right.$ EMDOGAIN $^{\mathbb{R}}$ ) for periodontal tissue regeneration in intrabony defects (Cochrane review). The Cochrane Library 2003; Issue 2. Oxford: Update Software.

4. Heijl L, Heden G, Svardstrom G et al. Enamel matrix derivative (EMDOGAIN ${ }^{\mathbb{R}}$ ) in the treatment of intrabony periodontal defects. J Clin Periodontol 1997; 24: 705-714.

Evidence-Based Dentistry (2003) 4, 64-65.

doi:10.1038/sj.ebd.6400195 